\title{
On Static and Radiative Space-Times
}

\author{
Helmut Friedrich \\ Max-Planck-Institut für Physik und Astrophysik, Institut für Astrophysik, Karl-Schwarzschild- \\ Straße 1, D-8046 Garching bei München, Federal Republic of Germany
}

\begin{abstract}
The conformal constraint equations on space-like hypersurfaces are discussed near points which represent either time-like or spatial infinity for an asymptotically flat solution of Einstein's vacuum field equations. In the case of time-like infinity a certain "radiativity condition," is derived which must be satisfied by the data at that point. The case of space-like infinity is analysed in detail for static space-times with non-vanishing mass. It is shown that the conformal structure implied here on a slice of constant Killing time, which extends analytically through infinity, satisfies at spatial infinity the radiativity condition. Thus to any static solution exists a certain "radiative solution" which has a smooth structure at past null infinity and is regular at past time-like infinity. A characterization of these solutions by their "free data" is given and non-symmetry properties are discussed.
\end{abstract}

\section{Introduction}

In article [9] the hyperboloidal initial value problem for Einstein's field equations was introduced. Here the prospective solution space-time is envisioned as having a smooth structure at future null infinity and data are given such that they represent the first and second fundamental forms on a space-like hypersurface which intersects null infinity in a space-like 2-sphere. In [12] has been shown that such "hyperboloidal initial data," if they are sufficiently close to Minkowskian hyperboloidal initial data, evolve into a solution of Einstein's equations which is future null geodesically complete, has in the future of the initial surface a smooth structure at null infinity, and is regular at future time-like infinity in the sense that in a suitable conformal extension of the solution a point $i^{+}$exists, representing future time-like infinity, such that the past directed null geodesics through $i^{+}$ generate future null infinity for the solution space-time.

This result reduces the question whether there exist non-trivial "purely radiative space-times," that is smooth solutions of Einstein's vacuum field equations which have a smooth and complete structure at past null infinity $\mathscr{I}^{-}$and future null infinity $\mathscr{I}^{+}$and which are regular at past time-like infinity $i^{-}$and future time-like infinity $i^{+}$, to the analysis of the behaviour of the solutions of the standard Cauchy problem near spatial infinity. In the following spatial infinity will be thought of 
as a point $i^{0}$ in a suitable conformal extension of the spacetime. The key problem (" $i{ }^{0}$-problem") now is to characterize those asymptotically flat Cauchy data which have an evolution "near $i^{0}$ " that allows "pieces" of smooth $\mathscr{I}^{-}, \mathscr{I}^{+}$near $i^{0}$, and to get control on the limit behaviour of $\mathscr{I}^{-}, \mathscr{I}^{+}$, if these data approach Minkowski data.

From the point of view of PDE theory one is asking here an extreme question. First of all one wants to solve a problem of a global nature, namely show for suitable data the existence of an evolution for which the outgoing null geodesics are complete. But on top of that one needs to derive estimates on the fall-off behaviour of the fields along these null geodesics which are so sharp and so precisely related to the structure of the initial data that they allow one to decide under which conditions on the data the solutions permit a smooth structure at null infinity.

The semiglobal results on the hyperboloidal initial value problem have been obtained by working entirely in the conformal picture. The use of the regular conformal field equations, which for positive conformal factor are equivalent to Einstein's field equations, allows, at least mathematically, a much broader view on the subject. To say that "the conformal fields propagate into the non-physical region" suddenly makes sense, since the regular conformal field equations are hyperbolic for any value of the conformal factor.

From this point of view one may look at the $i^{0}$-problem as follows. At the point $i^{0}$ the conformal fields which represent the conformal initial data on the hypersurface $S=\tilde{S} \cup\left\{i^{0}\right\}$, where $\tilde{S}$ is a Cauchy surface for the physical space-time, are partly non-smooth and partly even unbounded if the mass of the physical space-time is to be positive. In particular the rescaled Weyl tensor shows a very specific singular behaviour at $i^{0}$ (see e.g. Eq. (4.19) and the subsequent remarks). If we could inspect all possible (not necessarily unique) solutions of the regular conformal field equations pertaining to the given data, we could find one of the following possibilities to be true. Either the singularity at $i^{0}$ spreads by necessity along the characteristics of the equations in such a way that at null infinity (referring always to the physical space-time) no smooth structure can be defined, or there are solutions which extend in a sufficiently smooth way through null infinity and develop a (in a suitable sense) time-like singularity through $i^{0}$ in the non-physical region.

The first possibility appears very likely in view of the hyperbolic nature of the regular conformal field equations. However, there are available a few results, which will be discussed in the following, where the situation is understood exactly and in sufficient detail. They suggest that there may exist a large class of data for which the second possibility will occur.

The vacuum Bianchi identities for the Weyl tensor, rewritten in terms of the conformal space-time and the rescaled Weyl tensor, form part of the regular conformal field equations (see Eq. (2.5)). If the latter are linearized at the Einstein cosmos (say, to stay in the conformal picture) one obtains from Eq. (2.5) the "linear spin-2 equations" on the Einstein cosmos for a tensor field with the symmetries of the conformal Weyl tensor. On the Einstein cosmos we choose now an analytic space-like Cauchy surface $S$ and on $S$ a point $i^{0}$, which is thought of as representing spatial infinity for the conformally embedded Minkowski space. On $S \backslash i^{0}$ we 
prescribe analytic initial data for the linear spin-2 equations (which satisfy in particular the constraints implied on $S$ by those equations). At the point $i^{0}$ we require the data to become singular so that they reflect in an appropriate way the singular behaviour of the rescaled Weyl tensor referred to above. If one studies now the solutions of the linear spin-2 equations for this type of singular data, one finds that for a large class of data the singularity at $i^{0}$ does not spread along the null cone at $i^{0}$, which also represents the characteristic cone of the equations at $i^{0}$ and also $\mathscr{I}^{+}, \mathscr{I}^{-}$for the embedded Minkowski space. Instead the solutions extend smoothly as solutions of the spin-2 equations through the null cone into the future of $i^{0}$.

This fact has been known for a long time. However, it is surprising that the specific structural properties of the equations which are responsible for this peculiar behaviour of its solutions have never been identified and used in a direct constructive way. Consequently nothing is known about the possible implications of these properties in the nonlinear case.

The only general class of solutions of Einstein's equations for which the asymptotic behaviour is fairly well understood is provided by static or stationary space-times. Here it can be shown that the conformal fields extend smoothly through past and future null infinity as solutions of the regular conformal field equations. But also in this case our understanding follows not so much from a general insight into the specific propagation mechanism provided by the equations but rather from our knowledge about the exact form of the solutions and their behaviour at space-like infinity. Needed instead is a much more detailed understanding of the behaviour of the fields near $i^{0}$ together with a deeper insight into the effects of the constraint equations and the propagation equations and in particular into the "interaction" of these two sets of equations near the singularity at $i^{0}$.

Further progress into this direction is hampered to a large extent by the absence of sufficiently general illustrative examples. Exact solutions of Einstein's field equations are required for possible generalization which not only allow a smooth structure at null infinity but which have also a positive mass and non-vanishing radiation fields. The fact that such solutions have not been found yet has sometimes been regarded as an argument against the concept of asymptotic simplicity. It is well known, however, that the requirement of asymptotic simplicity together with the presence of more than one symmetry of the field, has very strong implications on the structure of the solutions (see the discussion in Sect. 5). If an exact solution of the type asked for above is to be found, it may at most allow one (space-like) Killing vector ficld. It is hard to come by such solutions and not many of them are available. Moreover, one will hardly feel tempted to embark on the complicated exploration of the asymptotic structure of a solution of low or no symmetry unless one has a clue for which particular solution to expect a positive result.

In view of all these difficulties and open problems it is of no use to be fascinated by generality. At present the most important and delicate problem seems to me to develop simplifying assumptions on the data, which allow one to analyse the $i^{0}$-problem in sufficient depth but which at the same time are general enough to 
identify those properties of the data and the equations which govern the asymptotic behaviour of the fields.

The results reported in this paper have been obtained in the course of an investigation of some of the problems outlined above. Though they are of interest in themself, the result appear to be particularly remarkable for a number of possibilities offered for further progress.

The conformal constraint equations, that is the constraint equations implied by the regular conformal field equations, are analysed on a space-like hypersurface $S$ near a point $i$ of $S$, which is distinguished by the requirement that at $i$ not only the conformal factor but also its differential vanishes, while its Hessian is not degenerate. For calculational convenience it is assumed that the first fundamental form $h$ on $S$ is analytic at $i$ and the second fundamental form of $S$ vanishes identically. Depending on further conditions on the data on $S$ the point $i$ pertains to different possible geometric and physical situations.

If the evolution of the data on $S$ by the regular conformal field equations is required to be smooth (here for simplicity in fact analytic) near $i$, the physical metric obtained on the time-like future $I^{+}(i)$ of $i$ in this evolution is a solution of Einstein's vacuum field equations with a smooth structure at past null infinity, for which the point $i$ represents a regular past time-like infinity $i^{-}$. Since such solutions are candidates for purely radiative space-times, they will be called "radiative space-times." It is found under the assumptions above that the conformal initial data on $S$ are determined uniquely, once the interior metric $h$ on $S$ is known. However, at the point $i$ the metric $h$, or more precisely the conformal structure defined by it, must satisfy a specific set of conditions (Theorem(3.1)), which will be called "radiativity condition" since any such metric determines a unique radiative space-time.

The other possible meaning of the point $i$ results from restricting attention to the points of the evolution of the data which are space-like related to $i$. Here again we have a solution of Einstein's field equations, for which $i$ now represents the point spatial infinity $i^{0}$. As mentioned before, the fields on $S$ will not be smooth at $i^{0}$ if this space-time is required to have non-vanishing mass. This situation is analysed in some detail in the particular case of static asymptotically flat solutions with non-vanishing mass, for which the 3-dimensional conformal structures implied on the slices of constant Killing time extend analytically to spatial infinity. It turns out that at spatial infinity these conformal structures all satisfy the radiativity condition (Theorem (4.4)).

This result gives rise to some interesting questions, has a number of direct consequences, and opens new prospects on the possibility to provide the desired "empirical material."

First of all we have a statement about the asymptotic behaviour of static fields. Whether the somewhat surprising fact that 3-dimensional metrics provided by static space-times satisfy the radiativity condition is accidental or of deeper significance is not clear yet. It should be of interest to translate this fact into a statement about the structure of the fields near spatial infinity $i^{0}$ in the 4dimensional space-time and relate it to the behaviour of the solution near null infinity. It is conceivable that a condition on the data like the radiativity condition 
is a prerequisite for the existence of a smooth structure at null infinity.

As an immediate consequence of Theorem (4.4) any static-time of the type considered here supplies a "radiative initial data set" and implies consequently the existence (Theorem (5.1)) of a radiative space-time (which, incidentally, entails the existence of hyperboloidal initial data, but still another argument is available for that). That the class of these solutions is indeed quite large, is indicated by a certain characterization of the static and thus also of the associated radiative space-times in terms of their "free" expansion coefficients (Theorem (4.2)). It should be of interest to relate this to the general discussion of radiative space-times in terms of their radiation fields on null infinity as given in [10]. For a simply way to translate results on radiative space-times from the conformal to the physical picture the reader may consult [13].

In constructing the radiative space-time as suggested by Theorem (5.1), the static Killing field is discarded. It is, however, conceivable that this Killing field is systematically replaced by another type of Killing field, which in the worst case may force the generators of past null infinity of the analytically extended radiative space-time to be incomplete. It is therefore shown in Theorem (5.3) that under simple conditions on the first few free expansion coefficients of the static space-time the associated radiative space-time allows no Killing field at all.

To come to a deeper understanding of the situation, one would like to generalize the investigation carried out here. It would be of interest to have available more general classes of metrics satisfying the radiativity condition and to extend the investigation to the case of non-vanishing second fundamental form. Such a generalization is necessary for the study of the evolution of the radiative initial data. Of some interest here is also the question whether stationary solutions have a similar behaviour at spatial infinity and determine in a similar way radiative space-times as the static solutions. A positive answer may lead to explicitly known radiative initial data with conformal Weyl tensors of the various Petrov types.

The most important problem, however, is to obtain more information on the evolution and possibly about the analytic extension of the radiative space-times, to answer questions about the completeness of past null infinity, the structure of spatial infinity, and eventually about the smoothness of future null infinity. Here it may be promising to employ a combination of general methods and techniques used in the study of exact solutions to take full advantage of the known information: the, possibly explicit, knowledge of the initial data, their symmetry, Petrov type, and the fact that they are derived from solutions of the static equations. Once the relation between static (respectively stationary) and radiative space-times has been established by using the regular conformal field equations, it may be useful to look at the situation from a different angle. The evolution of the radiative data by the regular conformal field equations determines not only the radiative solution in the future of the point $i$ but also in the set of points which are space-like related to $i$ an asymptotically flat solution of Einstein's vacuum field equations with smooth structure at null infinity and vanishing mass. If for specific data this space-time could be identified as one of the known exact solutions, the radiative space-time could be obtained by conformal and analytic extension.

If the overall structure of the radiative space-time is to be understood properly 
it may turn out necessary to study the situation from an even more general point of view. There are certain asymptotically flat static solutions, for which the metric induced on a slice of constant Killing time has two "spatial infinities" and a ring-like singularity [23]. Going to the associated radiative data, the spatial infinities become "past time-like infinities" $i_{1}^{-}, i_{2}^{-}$. The evolution of these data yields a solution of the regular conformal field equations near the initial surface, which contains a singularity and separated from it and from each other the sets $I^{+}\left(i_{1}^{-}\right), I^{+}\left(i_{2}^{-}\right)$on which two "physical" radiative space-times come into existence. In the transition from the static to the radiative data the latter acquire a reflection symmetry which would not be recognized if attention were focussed onto the neighbourhood of one of the points time-like infinity alone. The use of such additional properties of the overall picture may be decisive to find the answer to the crucial question: If the evolution of the radiative data develops further in time, does the singularity eventually penetrate into the radiative solutions or does it stay apart and possibly just "touches" them at their corresponding spatial infinities? However this may be, it is seen that the overall conformal picture offers quite new possibilities to think about a problem posed, though possibly not answered correctly, by Einstein [7]: Is a solution of the vacuum field equations generated necessarily by a matter source or a ("physical") space-time singularity?

\section{The Conformal Constraint Equations}

The object of the subsequent discussions is the construction of a 4-dimensional Lorentz-space with manifold $M$ and metric $g$ from data which are given on a 3-dimensional manifold $S$. The initial data surface $S$ is thought of as being smoothly embedded into $M$ as a space-like hypersurface. The metric $g$ will be obtained as part of a solution of the regular conformal field equations. In this section the constraint equations and a procedure to construct solutions to the constraint equations will be studied. Finally the structure and the meaning of these solutions is discussed for two different types of assumptions on their asymptotic behaviour. All calculations refer to the conformally rescaled space-time, which for the situations considered here provides in fact the only appropriate point of view.

It will be convenient to express all fields with respect to an orthonormal frame $e_{k}$ (indices $i, k, j, 1 \ldots$ will take values $0,1, \ldots, 3$, the summation convention is assumed), such that $g_{i k} \equiv g\left(e_{i}, e_{k}\right)=\operatorname{diag}(-1,1,1,1)$. In the following $(M, g)$ will be assumed time oriented and $e_{0}$ as future directed. We shall denote by $\nabla_{i}$ the covariant torsion free derivative defined by $g$ in the direction of $e_{i}$ and by $r_{j k l}^{i}, c_{j k l}^{i}, r_{j l}, r$ the curvature tensor, the conformal Weyl tensor, the Ricci tensor and the Ricci scalar of $g$, respectively. The regular conformal field equation forms a system of partial differential equations for a set of tensor fields which comprises besides the metric $g$ the "conformal factor" $\Omega$, its differential $\Sigma_{i}=\nabla_{i} \Omega$, the function $s=\frac{1}{4} \nabla_{i} \nabla^{i} \Omega$, the symmetric trace-free tensor $s_{i j}=\frac{1}{2}\left(r_{i j}-\frac{1}{4} r g_{i j}\right)$ and the rescaled Weyl tensor $d^{i}{ }_{j k l}=\Omega^{-1} c^{i}{ }_{j k l}$. The equations are given by

$$
\begin{aligned}
r_{i j k l} & =\Omega d_{i j k l}+2\left\{g_{i[k} s_{l] j}+g_{j[l} s_{k] i}\right\}+\frac{1}{6} r g_{i[k} g_{l] j}, \\
\nabla_{i} \Sigma_{j} & =-\Omega s_{i j}+s g_{i j},
\end{aligned}
$$




$$
\begin{gathered}
\nabla_{i} S=-\Sigma^{j} S_{j i}-\frac{1}{12} r \Sigma_{i}-\frac{1}{24} \Omega \nabla_{i} r, \\
\nabla_{k} s_{l j}-\nabla_{l} s_{k j}=\Sigma_{i} d^{i}{ }_{j k l}-\frac{1}{12} g_{j[l} \nabla_{k]} r, \\
\nabla_{i} d^{i}{ }_{j k l}=0, \\
6 \Omega S-3 \Sigma_{j} \Sigma^{j}+\frac{1}{4} \Omega^{2} r=0 .
\end{gathered}
$$

The function $r$ is considered here as an arbitrary smooth function of the space-time coordinates $x^{\mu}, \mu=0, \ldots, 3$.

In the region $\tilde{M}=\{x \in M \mid \Omega(x)>0\}$, Eqs. (2.1)-(2.6) are equivalent to Einstein's field equations $\operatorname{Ric}[\tilde{g}]=0$ for the "physical metric" $\tilde{g}=\Omega^{-2} g$. However, the equations generalize Einstein's equation by being meaningful also where $\Omega$ vanishes or becomes negative (in which case $\tilde{g}$ defines again a solution of Einstein's field equations!). This makes the equation particularly useful in the analysis of the asymptotic behaviour of solutions of Einstein's field equations, the set $\mathscr{I}=\{x \in M \mid \Omega(x)=0, d \Omega(x) \neq 0\}$ representing null conformal infinity for the solution $(M, g)$. In $[8,9,11,12]$ Eqs. (2.1)-(2.6) have been used to study the existence and properties of solutions to Einstein's equations which have a smooth structure at null conformal infinity and the reader is referred to those articles for a detailed discussion of the regular conformal field equations.

In this paper situations will be investigated where on the initial manifold $S$ is a point $i$ at which not only $\Omega$ but also its differential vanishes while the Hessian of $\Omega$ is non-degenerate. The first step of such an analysis requires the study of the properties of the fields on the initial surface $S$. In the following the "conformal constraint equations" implied by the system (2.1)-(2.6) on $S$ will be discussed and the procedure developed by Lichnerowicz, Choquet-Bruhat, and York [6] to provide solutions to the vacuum constraints is described in terms of the conformal fields.

Let the frame $e_{k}$ be chosen with $e_{0}$ normal to $S$, such that $e_{a}, a=1,2,3$, represents a frame on $S$. In the following tensor fields intrinsic to $S$ will be expressed with respect to this frame, indices $a, b, c, \ldots$ take values $1,2,3$, and the summation rule is assumed for these indices. The interior metric $h$ implied on $S$ by $g$ has components $h_{a b}=g_{a b}=\delta_{a b}$ and all index operations will be performed with this metric. Let $D_{a}$ denote the covariant torsion-free derivation in the direction of $e_{a}$ defined by $h$. In contrast to space-time curvature fields curvature derived from the 3-dimensional metric $h$, such as the curvature tensor $R_{b c d}^{a}$, the Ricci tensor $R_{a b}$, its trace-free part $S_{a b}$, its trace $R$ and the Bach tensor $B_{c a b}=D_{[a} R_{b] c}-\frac{1}{4} D_{[a} R h_{b] c}$ will be denoted by upper case letters. By $\chi_{a b}=g\left(\nabla_{a} e_{0}, e_{b}\right)$ will be denoted the second fundamental form of $S$. We shall consider $\Sigma_{a}=D_{a} \Omega, \Sigma \equiv \Sigma_{0}, s_{a} \equiv s_{a 0}, s_{a b}, d_{a b}=d_{a o b o}, d_{a b c}=d_{a o b c}$ as components of tensor fields on $S$ (shunting by this remark a lengthy discussion of a projection formalism). These fields have the algebraic properties

$$
\begin{aligned}
s_{a}^{a} & =s_{00}, \quad d_{a b}=d_{(a b)}, d_{a}^{a}=0, \quad d_{a c b}=-d_{a b c}, \quad d_{[a b c]}=0, \\
d_{a c}^{a} & =0, \quad d_{e a b c}=2\left\{h_{e[b} d_{c] a}+h_{a[c} d_{b] e}\right\} .
\end{aligned}
$$

The constraint equations implied by Eqs. (2.1)-(2.6) on $S$ are given by $[9,11]$

$$
R_{e a b c}=-2 \chi_{e[b} \chi_{c] a}+\Omega d_{e a b c}+2\left\{h_{e[b} s_{c] a}+h_{a[c} s_{b] e}\right\}+\frac{1}{6} r h_{e[b} h_{c] a},
$$




$$
\begin{aligned}
D_{b} \chi_{c a}-D_{c} \chi_{b a} & =\Omega d_{a b c}+2 h_{a[b} s_{c]}, \\
D_{a} \Sigma_{b} & =\Sigma \chi_{a b}-\Omega s_{a b}+s h_{a b}, \\
D_{a} \Sigma & =\Sigma^{c} \chi_{c a}-\Omega s_{a}, \\
D_{a} s & =\Sigma s_{a}-\Sigma^{c} s_{c a}-\frac{1}{12} r \Sigma_{a}-\frac{1}{24} \Omega D_{a} r, \\
D_{a} s_{b}-D_{b} s_{a} & =2 \chi_{[a}{ }^{c} s_{b] c}+\Sigma^{c} d_{c a b}, \\
D_{a} s_{b c}-D_{b} s_{a c} & =2 \chi_{c[a} s_{b]}+\Sigma d_{c a b}+\Sigma^{e} d_{e c a b}+\frac{1}{12} h_{c[a} D_{b]} r, \\
D^{c} d_{c a b} & =2 d_{c[a} \chi_{b]}^{c}, \\
D^{c} d_{a c} & =\chi^{c d} d_{c a d}, \\
0 & =6 \Omega s+3 \Sigma^{2}-3 \Sigma_{a} \Sigma^{a}+\frac{1}{4} \Omega^{2} r .
\end{aligned}
$$

Since in 3 dimensions the curvature tensor can always be expressed in terms of the Ricci-tensor, Eq. (2.7) is equivalent to

$$
R_{a b}=-\chi_{c}^{c} \chi_{a b}+\chi_{a}^{c} \chi_{c b}+\Omega d_{a b}+s_{a b}+\left(\frac{1}{6} r+s_{00}\right) h_{a b} .
$$

We shall now derive two equations which correspond to the Hamiltonian and the momentum constraint for the physical fields. Using Eq. (2.16) and the contracted forms of Eqs. (2.9), (2.17) we obtain

$$
2 \Omega \Delta \Omega-3 D_{a} \Omega D^{a} \Omega+\frac{1}{2} \Omega^{2} R=-3 \Sigma^{2}-\frac{1}{2} \Omega^{2}\left\{\left(\chi_{c}^{c}\right)^{2}-\chi^{a b} \chi_{a b}\right\}+2 \Omega \Sigma \chi_{c}^{c},
$$

where $\Delta=D_{a} D^{a}$ denotes the Laplacian. Contracting (2.8) and using (2.10) to eliminate $s_{a}$ yields

$$
D^{b}\left(\Omega^{-2} \chi_{b a}\right)=\Omega^{-2}\left\{D_{a}\left(\chi_{c}^{c}\right)-2 \Omega^{-1} D_{a} \Sigma\right\} .
$$

If $\tilde{h}_{a b}, \tilde{\chi}_{a b}$ denote the first and the second fundamental form on $S$ with respect to the physical metric (expressed in the frame $e_{a}$ ), we have the transformation law

$$
\chi_{a b}=\Omega\left(\tilde{\chi}_{a b}+\Sigma \tilde{h}_{a b}\right),
$$

which implies for the traces $\chi=h^{a b} \chi_{a b}, \tilde{\chi}=\tilde{h}^{a b} \tilde{\chi}_{a b}$, the transformation law

$$
\Omega \chi=\tilde{\chi}+3 \Sigma \text {. }
$$

We are free to choose $\Sigma$ on $S$. Therefore the assumption

$$
\chi_{c}^{c} \equiv 0, \quad \Sigma \equiv 0 \text { on } S,
$$

which will be made in the following just means that we require $S$ to be a maximal hypersurface for the physical as well as for the unphysical metric. With (2.20) Eqs. (2.18), (2.19) simplify to

$$
\begin{gathered}
\left(\Delta-\frac{1}{8} R\right) \theta=-\frac{1}{8} \chi^{a b} \chi_{a b} \theta \text { with } \theta=\Omega^{-1 / 2}, \\
D^{b}\left(\Omega^{-2} \chi_{b a}\right)=0 .
\end{gathered}
$$

A solution to the constraint equations (2.7)-(2.17) can now be constructed in the following way:

i) assume a metric $h$ is given on $S$ and $\psi_{a b}$ is a symmetric trace-free (with respect 
to $h)$ tensor which satisfies

$$
D^{a} \psi_{a b}=0 \text { on } S,
$$

ii) set $\chi_{a b}=\theta^{-4} \psi_{a b}$ in (2.21) and find a positive solution $\theta$ of

$$
\left(\Delta-\frac{1}{8} R\right) \theta=-\frac{1}{8} \psi^{a b} \psi_{a b} \theta^{-7},
$$

set $\Omega=\theta^{-2}, \chi_{a b}=\Omega^{2} \psi_{a b}$,

iii) solve the contracted form of Eq. (2.8) for $s_{a}$, Eq. (2.16) for $s$, Eq. (2.9) for $s_{a b}$, Eq. (2.8) for $d_{a b c}$ and Eq. (2.17) for $d_{a b}$, and calculate subsequently all fields from $h_{a b}, \chi_{a b}, \Omega$. For the particular choice $r \equiv 0$ on $S$ it follows from a system of differential identities given in [9] that in fact all the equations (2.7)-(2.17) are satisfied now. For arbitrary $r$ this follows in a similar way.

To avoid the lengthy algebraic calculations in this procedure, we shall assume in the following that

$$
\chi_{a b} \equiv 0 \text { on } S
$$

which implies by (2.10), (2.8),

$$
s_{a} \equiv 0, \quad d_{a b c} \equiv 0 \text { on } S .
$$

A solution of the constraint equations which satisfies (2.20), (2.23), (2.24) and thus consists of the fields

$$
\begin{aligned}
& h_{a b}, \Omega, s=\frac{1}{3} \Delta \Omega+\frac{1}{24} \Omega(2 R-r) \\
& S_{a b}=-\Omega^{-1}\left(D_{a} D_{b} \Omega-\frac{1}{3} \Delta \Omega h_{a b}\right)+\frac{1}{24}(2 R-r) h_{a b} \\
& d_{a b}=\Omega^{-2}\left(D_{a} D_{b} \Omega-\frac{1}{3} \Delta \Omega h_{a b}\right)+\Omega^{-1} S_{a b}
\end{aligned}
$$

will be called a "time symmetric initial data set."

In solving (2.21) one will in general assume some boundary conditions to be satisfied. We choose a point $i \in S$, which will represent (spatial, say) infinity of the physical fields. To simplify the following considerations we assume that the metric $h$ is smooth everywhere on $S$ and real analytic in some neighbourhood $V$ of $i$ in $S$. Suppose that $x^{\alpha}, \alpha=1,2,3$ is a system of normal coordinates for $h$ centered at the point $i$, set $x^{2}=\Sigma\left(x^{\alpha}\right)^{2},|x|=\left(x^{2}\right)^{1 / 2}, y^{\alpha}=|y|^{-2} x^{\alpha},|y|=\left(y^{2}\right)^{1 / 2}$, etc. Let $\tilde{h}_{\alpha \beta}$ be the expression of the metric $h=\Omega^{-2} h$ in the coordinates $y^{\alpha}$. In order that $i$ represents (spatial) infinity for the metric $\tilde{h}$ and the "usual" fall-off conditions

$$
\tilde{h}_{\alpha \beta}-\delta_{\alpha \beta}=0\left(\frac{1}{|y|}\right) \text { for }|y| \rightarrow \infty
$$

are satisfied, we need to have

$$
\theta=\Omega^{-1 / 2}=0\left(\frac{1}{|x|}\right) \text { for }|x| \rightarrow 0 .
$$

The solutions of the conformally invariant Laplace equation

$$
\left(\Delta-\frac{1}{8} R\right) \theta=0
$$


on $S \backslash i$, which satisfy $\lim (|x| \theta)=1$, have near $i$ the following structure (see e.g. [14]) $|x| \rightarrow 0$

$$
\theta=\frac{U}{|x|}+W
$$

with functions $U, W$ which are real analytic at $i$ and such that

i) $W$ is a smooth solution of (2.26),

ii) $U=\sum_{k=0}^{\infty} U_{k}|x|^{2 k}, U_{k}$ real analytic at $i$,

$$
U_{0}=1+0\left(|x|^{2}\right) \text { for }|x| \rightarrow 0 .
$$

The function $\Omega$ is thus given by

$$
\Omega=\frac{x^{2}}{(U+|x| W)^{2}}
$$

The mass of the metric $\tilde{h}$ is given by $m=2 W(i)$. If $S$ is the 3 -dimensional sphere $S^{3}$ and $\Omega$ required to be smooth and positive everywhere on $S^{3} \backslash i$, we have $W(i)>0$ by the positive mass theorem unless $\tilde{h}$ is flat [20]. Therefore one might be tempted to discard conformal factors of the form (2.27), defined near $i$ and such that $W$ vanishes at $i$ up to a certain order or altogether, as physically uninteresting. But this is not necessarily true.

Assume that $\Omega$ as well as the initial data set (2.25) is smooth in a neighbourhood of $i$. Then the regular conformal field equations determine an evolution $(M, g, \Omega)$ of these data, which defines in fact two different solutions of Einstein's vacuum field equations. Denote by $I^{+}(i)$ respectively $I^{-}(i)$ the time-like future respectively past of $i$ in $(M, g)$ and by $N_{i}^{+}$respectively $N_{i}^{-}$the cone generated by the null geodesics which leave the point $i$ into the future respectively into the past. From (2.27) and the properties of $U$ it follows that $\Omega$ has a non-degenerate critical point at $i$. This and Eqs. (2.1)-(2.6) imply (possibly after restricting $M$ suitably) that $i$ is the only critical point of $\Omega$ and that the zero set of $\Omega$ coincides with the set $N_{i}^{-} \cup N_{i}{ }^{+}$(see [12] for the details of the argument). On the set $M \backslash\left\{I^{+}(i) \cup N_{i}{ }^{+} \cup I^{-}(i) \cup N_{i}{ }^{-}\right\}$the metric $\tilde{g}=\Omega^{-2} g$ is a solution of Einstein's field equations for which $i$ represents spatial infinity and which has vanishing mass. However, on the set $I^{+}(i)$ the metric $\tilde{g}$ also represents a solution of Einstein's vacuum field equations, for which the point $i$ now represents past time-like infinity and the set $\mathscr{I}^{-}=N_{i} \backslash i$ past null infinity. We shall refer to the fact that $\mathscr{I}^{-}$can be obtained as a null cone of a point in the conformal extension as to the "regularity of the point past time-like infinity." A solution of Einstein's field equation, which is regular at past timelike infinity, is a candidate for a space-time representing pure selfinteracting gravitational radiation [10], and therefore of considerable physical interest. In the following we shall be interested to provide time-symmetric initial data sets which lead to smooth solutions of the type $\left(I^{+}(i), \tilde{g}\right)$.

\section{The Radiativity Condition}

If we assume $W=0$ in the expression (2.27) the function $\Omega$ will be real analytic. However the fields $s_{a b}, d_{a b}$ will in general not show the same degree of smoothness. 
It is the purpose of the present section to show that the metric $h$ must behave in a very specific way near $i$ to ensure the analyticity of the data (2.25).

In the following only conformal factors of the form (2.27) for which $W$ vanishes identically will be considered. In a certain neighbourhood of $i$, which we assume to coincide with $V$, the function $\Omega$ is real analytic and satisfies

$$
\begin{gathered}
2 \Omega \Delta \Omega=3 D^{2} \Omega D_{a} \Omega-\frac{1}{2} \Omega^{2} R \\
\left.\Omega\right|_{i}=0,\left.\quad D_{a} \Omega\right|_{i}=0,\left.\quad D_{a} D_{b} \Omega\right|_{i}=\left.2 h_{a b}\right|_{i} .
\end{gathered}
$$

The conformal factor is fixed uniquely by (3.1), (3.2) and the requirement of analyticity.

We shall now derive conditions on the metric $h$, which ensure that the fields $s_{a b}, d_{a b}$ given in (2.25) will also be analytic at $i$. Allow the normal coordinates $x^{\alpha}$ to take values in a neighbourhood $V_{\mathbb{C}}$ of the origin of $\mathbb{C}^{3}$ which is chosen such that the fields $h, e_{a}, \Omega$ extend by analyticity to holomorphic fields on $V_{\mathbb{C}}$. Furthermore, we assume that the point $i=\left\{x^{\alpha}=0\right\}$ is the only critical point of $\Omega$ in $V_{\mathbb{C}}$ and that the null cone generated by the complex null geodesics of $h$ through $i$ is given by the analytic set $N_{\mathbb{C}}=\left\{x^{\alpha} \in V_{\mathbb{C}} \mid \sum_{\alpha=1}\left(x^{\alpha}\right)^{2}=0\right\}$. It follows from (2.27) or from (3.1), (3.2) that the zero set of $\Omega$ in $V_{\mathbb{C}}$ coincides with $N_{\mathbb{C}}$. Since $N_{\mathbb{C}}$ is irreducible and $\Omega$ coincides up to a non-vanishing factor with $x^{2}$ we have the following fact, which will be used repeatedly: If $f$ is a holomorphic function on $V_{\sigma}$, with $\left.f\right|_{N_{\odot}} \equiv 0$, then there exists a holomorphic function $g$, defined on a neighbourhood of $i$ in $V_{\mathbb{C}}$, such that $f=\Omega g$ (see e.g. [17]).

By (2.25) the field $d_{a b}$ extends to a holomorphic function on $V_{\mathbb{C}} \backslash N_{\mathbb{C}}$ such that

$$
\Omega^{2} d_{a b}=D_{a} D_{b} \Omega-\frac{1}{3} \Delta \Omega h_{a b}+\Omega S_{a b} .
$$

If $d_{a b}$ were holomorphic on $V_{\mathbb{C}}$, this equation would imply

$$
\left.\left(D_{c}\left\{D_{a} D_{b} \Omega-\frac{1}{3} \Delta \Omega h_{a b}+\Omega S_{a b}\right\}\right)\right|_{N_{\mathbb{C}}} \equiv 0 .
$$

Conversely the relation (3.4) implies the existence of holomorphic functions $f_{a b c}$ near $i$ such that

$$
D_{c}\left\{D_{a} D_{b} \Omega-\frac{1}{3} \Delta \Omega h_{a b}+\Omega S_{a b}\right\}=\Omega f_{a b c} .
$$

Transvecting this equation with $D^{c} \Omega$ and restricting to a null generator of $N_{\mathbb{C}}$ yields an ordinary differential equation for the term in curly brackets, which allows one to conclude that this term is constant along the generators and thus vanishes on $N_{\mathbb{C}}$, since it vanishes at $i$. Consequently we have a relation

$$
D_{a} D_{b} \Omega-\frac{1}{3} \Delta \Omega h_{a b}+\Omega S_{a b}=\Omega f_{a b}
$$

near $i$ with holomorphic functions $f_{a b}$. Differentiating this equation and comparing the result with (3.5) gives $\left.\left(D_{c} \Omega f_{a b}\right)\right|_{N_{c}} \equiv 0$. Since $D_{c} \Omega \neq 0$ in $V_{c} \backslash$, it follows that $f_{a b}=\Omega \tilde{d}_{a b}$ with some holomorphic functions $\tilde{d}_{a b}$ which coincide in $V_{\mathbb{C}} \backslash N_{\mathbb{C}}$ near $i$ with $d_{a b}$.

Thus the analyticity of $d_{a b}$ near $i$ is equivalent to the relation (3.4). Since the tensor $\Omega^{2} d_{a b}$ given by (3.3) is a conformal density condition (3.4) is in fact a condition on the conformal class of $h$. From (2.25) if follows immediately that $s_{a b}$ is analytic, if $d_{a b}$ is analytic. 
There is another interesting relation which imposes conditions on the conformal class of $h$, if $d_{a b}$ is analytic. We set $L_{a b}=R_{a b}-\frac{1}{4} R h_{a b}$ and write Eq. (3.3) in the form

$$
0=\Omega L_{a b}+D_{a} D_{b} \Omega-\frac{1}{3} h_{a b}\left(\Delta \Omega+\frac{1}{4} R \Omega\right)-\Omega^{2} d_{a b}
$$

in $V \backslash i$. Applying the operator $D_{c}$ and contracting on $a$ and $c$ gives

$$
D_{b} \Delta \Omega+\frac{1}{4} D_{b}(R \Omega)+3 D^{c} \Omega L_{c b}=3 \Omega D^{c} \Omega d_{c b}
$$

if the constraint Eq. (2.15) is taken into account. Applying again $D_{c}$ to (3.6) and antisymmetrizing gives

$$
\begin{aligned}
0= & \Omega\left\{D_{c} L_{a b}-D_{a} L_{c b}-\Omega\left(D_{c} d_{a b}-D_{a} d_{c b}\right)-2\left(D_{c} \Omega d_{a b}-D_{a} \Omega d_{c b}\right)\right\} \\
& -\frac{1}{3} g_{a b}\left\{D_{c} \Delta \Omega+\frac{1}{4} D_{c}(R \Omega)+3 D^{d} \Omega L_{d c}\right\} \\
& +\frac{1}{3} g_{b c}\left\{D_{a} \Delta \Omega+\frac{1}{4} D_{a}(R \Omega)+3 D^{d} \Omega L_{d a}\right\},
\end{aligned}
$$

where the expression of $R_{a b c d}$ in terms of $L_{a b}$, which holds in 3 dimensions, has been used. Using (3.7) to simplify yields the following expression for the conformally invariant $\mathrm{Bach}-$ tensor:

$$
B_{c a b}=D_{[a} L_{b] c}=2 D_{[a} \Omega d_{b] c}+D^{d} \Omega d_{d[a} h_{b] c}+\Omega D_{[a} d_{b] c} .
$$

It is worthwhile pointing out that in the derivation of this relation on $V \backslash i$ the assumption that $\Omega$ be analytic at $i$ has not been used. Thus (3.8) is true in $V \backslash i$ for any choice of $W$ in the expression (2.27) of $\Omega$. It is remarkable that the particular combination of $\Omega, d_{a b}$ and their derivations on the right of (3.8) is independent of $W$ and extends to an analytic function at $i$ even if $d_{a b}$ is not analytic at $i$.

The consequences of the relations (3.4) and (3.8) are worked out most easily in terms of space spinors (defined with respect to the normal $e_{0}$ on $S$ ) [21]. Not only is this spinor formalism particularly well adapted to the complex null-geometry, but also has the operator

$$
T_{a_{1} \ldots a_{q}} \mapsto C_{a_{1} \ldots a_{p}}\left(T_{a_{1} \ldots a_{p} a_{p}+1 \ldots a_{q}}\right),
$$

which maps the tensor $T_{a_{1} \ldots a_{q}}$ on $S$ onto its part which is symmetric and trace-free in the indices $a_{1}, \ldots, a_{p},(p \leqq q)$, a particularly simple representation in the spinor formalism.

If $T_{A_{1} B_{1} \ldots A_{q} B_{q}}=T_{\left(A_{1} B_{1}\right) \ldots\left(A_{q} B_{q}\right)}$ is the space spinor representation of the tensor $T$, then the tensor $C_{p} T$ is represented simply by $T_{\left(A_{1} B_{1} \cdots A_{p} B_{p}\right) A_{p+1}, B_{p+1}} A_{q} B_{q}$. As we shall see, the operator $C$ is crucial for expressing the condition that certain functions vanish on the complex null cone in terms of the real structures we started with.

If $B_{A B C D}$ is the space spinor representation of the tensor $B_{a b}=\frac{1}{2} B_{a e f} \varepsilon^{e f}{ }_{b}$ (where $\varepsilon_{123}=1, \varepsilon_{a b c}=\varepsilon_{[a b c]}$, which contains all the information on the Bach tensor and is symmetric and trace-free, then Eq. (3.8) takes in spatial spinor notation the form

$$
B_{A B C D}=2 D_{E(A} \Omega d_{B C D)}{ }^{E}+\Omega D_{E(A} d_{B C D)}{ }^{E} .
$$

Assume now that $d_{A B C D}$ is analytic at $i$ and all fields are extended by analyticity to $V_{\mathbb{C}}$. Let $\kappa^{A}$ be a spinor at $i$, denote by $\mathbb{C} \ni u \mapsto \kappa(u) \in N_{\mathbb{C}}$ the null geodesic through $i=\kappa(0)$ with tangent vector $\kappa^{A} \kappa^{B}$ at $i$ and extend $\kappa^{A}$ along $\kappa(u)$ by parallel transport. Then $\kappa^{A} D_{A B} \Omega=0$ along $\kappa(u)$ and (3.9) yields

$$
\kappa^{A} \kappa^{B} \kappa^{C} \kappa^{D} B_{A B C D}(\kappa(u)) \equiv 0 \text { for } u \text { near } 0
$$


Applying repeatedly the operator $\kappa^{A} \kappa^{B} D_{A B}$ to this equation, evaluating at $i$ and taking into account that $\kappa^{A}$ was chosen arbitrarily, we obtain a condition in terms of structures on $V$, which is equivalent to (3.10)

$$
D_{\left(A_{n} B_{n} \ldots A_{1} B_{1}\right.} B_{A B C D)}(i)=0, \quad n=0,1,2, \ldots
$$

This is a necessary condition on the conformal class of $h$ for $d_{a b}$ to be analytic at $i$. In tensor language it is written

$$
C_{a_{n} \ldots a_{1} a b}\left(D_{a_{n} \ldots} D_{a_{1}} B_{a b}\right)(i)=0 \quad n=0,1,2, \ldots
$$

Translating in the same way (3.4) into an equivalent condition on structures on $V$ we arrive at

Theorem (3.1). Assume that the metric $h$ and the conformal factor $\Omega$ satisfying (3.1), (3.2) are analytic on a neighbourhood $V$ of $i \in S$. Then the time symmetric initial data set (2.25) is analytic on $V$ if and only if $h$ is such that the "radiativity condition"

$$
C_{a_{n} \ldots a_{1}}\left\{D_{a_{n} \ldots} D_{a_{1}} D_{c}\left(D_{a} D_{h} \Omega-\frac{1}{3} \Delta \Omega h_{a b}-\Omega S_{a b}\right)(i)\right\}=0, \quad n=0,1,2, \ldots
$$

is satisfied. If the radiativity condition holds, the Bach tensor of h satisfies the relation (3.12).

Remarks. The condition (3.13) appears much stronger than (3.12). It would be interesting to understand to what extent this is true.

As long as we are interested in analytic data and analytic solutions to the propagation equations, the conditions (3.4) and (3.13) may be used alternatively. If we want to restrict, however, the assumptions on the data and the evolution to the $C^{k}$-case, only a formulation of the radiativity condition of the type (3.13) (with $n$ up to a suitable order) is appropriate.

We shall call a time symmetric initial data set (2.25) which is analytic at $i$ a "radiative initial data set."

\section{On Static Space-Times}

The most important question now is whether there exist non-trivial metrics for which the condition (3.13) is satisfied. After some unsuccessful attempts to set up a differential system which would supply as solutions metrics satisfying the radiativity condition, it turned out that such a system has been available for some time and is, somewhat surprisingly, provided by the static field equations and their conformal extension as discussed by Beig and Simon [3].

If a space-time with physical metric $\tilde{g}$ admits a time-like hypersurfaceorthogonal Killing vector field, i.e. if $\tilde{g}$ is static, one can choose coordinates $x^{0}, x^{\alpha}$, $\alpha=1,2,3$, such that the metric takes the form

$$
\tilde{g}=-e^{2 U}\left(d x^{0}\right)^{2}+e^{-2 U} \hat{h}_{\alpha \beta} d x^{\alpha} d x^{\beta}
$$

with $x^{0}$-independent functions $U, \hat{h}_{\alpha \beta}$. Einstein's vacuum field equations for such a metric reduce to the quasilinear (in a suitable gauge elliptic) system of equations

$$
\begin{aligned}
& \hat{R}_{\alpha \beta}=2 \hat{D}_{\alpha} U \hat{D}_{\beta} U, \\
& \hat{\Delta} U=0
\end{aligned}
$$


for the function $U$ and the Riemannian metric $\hat{h}$. The covariant operator $\hat{D}$, the Laplacian $\hat{\Delta}$ and the Ricci-tensor $\hat{R}_{\alpha \beta}$ are defined here with respect to $\hat{h}$. In the following solutions of these equations will be considered which exist with $U \neq 0$ on some 3 -dimensional manifold $\hat{S}$ (representing a slice $\left\{x^{0}=\right.$ const $\}$ ) which is diffeomorphic to $\mathbb{R}^{3}$ minus a closed non-empty ball. The solution will be required to be asymptotically flat and we shall be concerned mainly with its behaviour near spatial infinity (with respect to $(\hat{h}, \hat{S})$ ). This behaviour has been analysed in considerable detail. Beig and Simon [3] showed for asymptotically flat solutions of (4.1), (4.2) with non-vanishing mass $M_{0}$ that the rescaled metric

$$
h_{\alpha \beta}=\omega^{2} \hat{h}_{\alpha \beta} \text { with } \omega=M_{0}^{-2} U^{2}, \quad M_{0} \neq 0,
$$

if expressed in suitable coordinates, extends to a real analytic metric on a manifold $S=\widetilde{S} \cup\{i\}$, which is obtained by attaching a point $i$ (representing spatial infinity for $\hat{h}$ ) to $\widetilde{S}$ such that $S$ is diffeomorphic to an open ball in $\mathbb{R}^{3}$. The basic step in their analysis was the derivation of a quasilinear elliptic system of differential equations, implied by (4.1), (4.2) for the conformal fields. The analyticity of the solutions of this system then followed from weak smoothness assumptions on the extension of $h_{\alpha \beta}, \omega$ to $S$ and from standard results on elliptic equations. The argument will not be repeated here and the equations for the conformal fields will be written in a form suitable to the following discussion. Furthermore, the analyticity of the solutions at $i$ will be assumed throughout.

We express again all fields in an orthonormal frame $e_{a}, a=1,2,3$, for $h$ on $S$. The conformal-static equations form a system of partial differential equations for the metric $h$, a symmetric trace-free tensor $S_{a b}$ and functions $\omega, t, R$. If $R_{a b}$ denotes the Ricci-tensor of $h$, the equations are given by

$$
\begin{aligned}
R_{a b} & =S_{a b}+\frac{1}{3} R h_{a b}, \\
D_{a} D_{b} \omega & =t h_{a b}-\omega R_{a b}+\frac{M_{0}^{2}}{2} D_{a} \omega D_{b} \omega, \\
D_{a} t & =D^{d} \omega\left(\frac{2}{3} R h_{d a}-S_{d a}\right), \\
D_{a} R & =M_{0}^{2} D^{d} \omega\left(\frac{2}{3} R h_{d a}-S_{d a}\right), \\
D_{[c} S_{a] b} & =M_{0}^{2} D^{d} \omega\left(\frac{1}{3} S_{d[c} h_{a] b}-\frac{1}{2} S_{b[c} h_{a] d}-\frac{R}{18} h_{d[c} h_{a] b}\right),
\end{aligned}
$$

and thus unknowns are required to satisfy the conditions

$$
\omega=0, \quad D_{a} \omega=0, \quad t=2, \quad R=M_{0}{ }^{2} t \text { at the point } i \in S
$$

in order that the physical metric $e^{-2 U} \hat{h}_{\alpha \beta}$ satisfy the asymptotic flatness conditions at infinity. For a discussion of assumptions (4.9) the reader may consult [3]. Before explaining how Eqs. (4.5)-(4.8) are obtained it is convenient to note a few consequences.

Lemma (4.1). A smooth solution of (4.4)-(4.9) also satisfies

$$
\begin{aligned}
\left.D_{a} D_{b} \omega\right|_{i} & =\left.2 h_{a b}\right|_{i}, \\
R & =M_{0}{ }^{2} t,
\end{aligned}
$$




$$
\begin{gathered}
R=\frac{M_{0}^{2}}{3} \Delta \omega, \\
\omega R=\frac{M_{0}^{2}}{2} D_{a} \omega D^{a} \omega, \\
2 \omega \Delta \omega=3 D_{a} \omega D^{a} \omega .
\end{gathered}
$$

Evaluation of (4.5) at $i$ gives in view of (4.9) Eq. (4.10). Equation (4.11) follows immediately by comparing (4.6), (4.7) and noting (4.9). Applying the operator $D_{c}$ to (4.5), commuting $D_{c}, D_{a}$ on the left, contracting with respect to indices $b, c$ and using (4.4)--(4.7) to simplify, we obtain the equation

$$
D_{a}(\Delta \omega-3 t)=M_{0}^{2} D_{a} \omega(\Delta \omega-3 t),
$$

which implies because of (4.10) that $t \equiv \frac{1}{3} \Delta \omega$ and by (4.11) also (4.12). By contraction of (4.5), (4.13) follows now, which together with (4.12) implies (4.14).

We see that in fact either (4.6) or (4.7) is redundant if we assume (4.11). However in some manipulations it is convenient to make a formal distinction between $t \equiv \frac{1}{3} \Delta \omega$ and $R$.

On a neighbourhood $V$ of $i$ such that on $V \backslash i$ the function $\omega$ is positive the equivalence of Eqs. (4.1), (4.2) with (4.4)-(4.8), if (4.9) is assumed, can be seen as follows. Equation (4.14) is just Eq. (4.2) expressed in terms of $\omega$ and $h$. Using (4.14), Eq. (4.5) is obtained by writing Eq. (4.1) in terms of $\omega$ and $h$ and introducing the notation $t=\frac{1}{3} \Delta \omega$. Equation (4.6) follows from (4.5) by taking a covariant derivative, commuting operators, contracting and observing (4.5) to simplify again. Equations (4.6), (4.7) are identical in view of (4.9) and Eq. (4.8) follows from (4.5) by taking a covariant derivative and antisymmetrizing.

For various purposes it is convenient to have available the characterization of the analytic solutions of (4.4)-(4.9), which is given in the following theorem.

Assume $h$ is analytic near $i, x^{\alpha}$ are normal coordinates for $h$ centered at $i$, and the orthonormal frame $e_{a}$ for $h$ near $i$ is parallel propagated along the geodesics through $i$ and such that $e_{a}=e_{a}{ }^{\alpha} \partial / \partial x^{\alpha}$ with $e^{\alpha}{ }_{a}=\delta^{\alpha}{ }_{a}$ at $i$. We write $x^{a}=\delta^{a}{ }_{\alpha} x^{\alpha}$. If $T$ is an analytic tensor field defined near $i$, it is well known that its components $T_{a_{1} \ldots a_{p}}^{b_{1} \ldots b_{q}}$ with respect to the frame $e_{a}$ can be obtained near $i$ as a convergent power series of the following form:

$$
T_{a_{1} \ldots a_{p}}^{b_{1} \ldots b_{q}}=\sum_{k \in \mathbb{N}} \frac{1}{k !} x^{c_{k}} \cdots x^{c_{1}}\left(D_{c_{k}} \cdots D_{c_{1}} T_{a_{1} \ldots a_{p}}^{b_{1} \ldots b_{q}}(i)\right)
$$

Theorem (4.2). A sequence $\sigma_{a_{1} \ldots a_{p}}, p \in \mathbb{N}, p \geqq 2$, of symmetric trace-free tensors at $i$ determines a unique formal-expansion type solution of (4.4)-(4.9), with expansions of $\omega, t, R, S_{a b}$ of the form (4.15), such that the coefficients of the expansion of $S_{a b}$ satisfy

$$
C_{a_{1} \ldots a_{p}}\left(D_{a_{p}} \ldots D_{a_{3}} S_{a_{2} a_{1}}(i)\right)=\sigma_{a_{1} \ldots a_{p}}, \quad p=2,3, \ldots
$$

Writing Eqs. (4.4)-(4.8) in the space spinor formalism, where in particular (4.8) takes the form

$$
D^{E}{ }_{A} S_{B C D E}=\frac{M_{0}^{2}}{4} D^{E F} \omega\left\{\varepsilon_{A(B} S_{C D) E F}-\varepsilon_{A E} S_{B C D F}-\varepsilon_{E(B} S_{C D) A F}\right\}-\frac{M_{0}{ }^{2}}{12} R \varepsilon_{A(B} D_{C D)} \omega .
$$


Therefore (4.2) is obtained as an immediate application of the theory of "exact set of fields" discussed in [18]. If a formal expansion of the curvature fields $S_{a b}, R$ is given, a formal expansion of the frame coefficients $e^{\alpha}{ }_{a}$ and the connection coefficients with respect to $e_{a}$ can be obtained from the well known expression of the structure equations in geodesic polar coordinates.

In the case that the formal expansions considered in Theorem (4.2) represent in fact convergent power series and thus define an analytic solution of (4.4)-(4.9), we may extend again the field $S_{A B C D}$ by analyticity to some neighbourhood of the origin of $\mathbb{C}^{3}$. Using the notation employed in (3.10), we see from Theorem (4.2) that an analytic solution of (4.4)-(4.9) is determined uniquely by the function

$$
\kappa^{A} \mapsto \kappa^{A} \kappa^{B} \kappa^{C} \kappa^{D} S_{A B C D}(\kappa(1)),
$$

which may be considered as a function on the complex null cone through $i$.

The content of Theorem (4.2) is not entirely new. A characterization of the solutions of (4.1), (4.2) with non-vanishing mass in terms of "multipole moments" [3] has been given before. These moments have been introduced because of their "nice" transformation behaviour under conformal rescalings [15]. They are related in a one-to-one though non-linear fashion to the quantities $M_{0}$ and $C\left(D_{a_{p}} \ldots D_{a_{3}} S_{a_{2} a_{1}}(i)\right)$. Thus both sets of data are equivalent. It may be pointed out however, that the use of the theory of exact sets of fields not only shows that the static solutions are characterized uniquely by the multipole moments but also that these moments are algebraically independent. Moreover, it appears that the characterization given here is more amenable to a convergence proof. Growth conditions on the $\sigma_{a_{1} \ldots a_{q}}$ which ensure convergence of the series considered in Theorem (4.2) will be considered elsewhere. There are sufficiently many exact solutions of (4.4)-(4.9) available such that the following results are of interest. Moreover, the existence of a large class of asymptotically flat static solutions has been established recently from quite a different point of view [19].

Any solution of (4.4)-(4.9) provides a time symmetric initial data set of the form (2.25) with metric $h$ and conformal factor $\Omega_{s}=\omega e^{-M_{0} \sqrt{ } \omega}$ (where the minus sign is introduced to have a positive mass for the physical fields if $M_{0}>0$ ). However, from the same solution other initial data sets can be derived.

Theorem (4.3). Suppose the function $\omega$ and the metric $h$ are obtained as part of an analytic solution of (4.4)-(4.9). Then all solutions (3.1), (3.2) of the form $\Omega=\Omega(\omega)$ are given by

$$
\Omega=\omega\left\{a \cosh \left(\frac{M_{0}}{2} \sqrt{\omega}\right)+b \sinh \left(\frac{M_{0}}{2} \sqrt{\omega}\right)\right\}^{-2}
$$

with suitable real numbers $a, b$. In particular the unique analytic solution of (3.1), (3.2) is given by

$$
\Omega_{A}=\omega\left\{\cosh \left(\frac{M_{0}}{2} \sqrt{\omega}\right)\right\}^{-2} .
$$

Setting $\Omega=\Omega(\omega)$ in Eq. (3.1) and writing $\Omega^{\prime}=d \Omega / d \omega$ etc, gives in view of (4.4)-(4.6) the ordinary differential equation 


$$
2 \omega \Omega \Omega^{\prime \prime}+3 \Omega \Omega^{\prime}-3 \omega\left(\Omega^{\prime}\right)^{2}+\frac{M_{0}^{2}}{4} \Omega^{2}=0
$$

It can be checked immediately that (4.16) is the general not identically vanishing solution of this equation. Analyticity of (4.16) requires $b=0$, conditions (3.2) imply $a=1$.

However, the form (4.17) of the analytic conformal factor may be deduced without solving the complicated equation (4.18). By (2.27) we must have

$$
\Omega_{S}{ }^{-1 / 2}=\Omega_{A}{ }^{-1 / 2}+W
$$

with some analytic solution $W$ of (2.26). With $\Omega_{S}=\omega e^{-M_{0 \vee} \omega}$ this can be rewritten

$$
W-\frac{1}{\sqrt{\omega}} \sinh \left(\frac{M_{0}}{2} \sqrt{\omega}\right)=\frac{1}{\sqrt{\omega}}\left\{\cosh \left(\frac{M_{0}}{2} \sqrt{\omega}\right)-\sqrt{\frac{\omega}{\Omega_{A}}}\right\} .
$$

Extending $\omega, \Omega_{A}$ by analyticity to a neighbourhood of the origin in $\mathbb{C}^{3}$, we find from (4.9), (4.10), (4.14) and from (3.1), (3.2) that the zero sets of $\omega$ and $\Omega_{A}$ coincide near $i$ and that the term in curly brackets on the right extends to an analytic function at $i$. Similarly the term on the left extends to an analytic function at $i$. Because of the factor $\omega^{-1 / 2}$ on the right this can only be the case if $W=\omega^{-1 / 2} \sinh \left(\left(M_{0} / 2\right) \sqrt{\omega}\right)$ and $\Omega_{A}$ is given by (4.17).

Having at our disposal the function $\Omega_{A}$, we can show

Theorem (4.4). Suppose the metric $h$ is obtained as part of an analytic solution of the conformal static equations (4.4)-(4.9). Then it satisfies the radiativity condition (3.13) at the point $i=\{\omega=0\}$.

Remarks. Since only a particular application of this result will be discussed in the next section, it may be emphasized here that Theorem (4.4) is first of all a statement about the structure of static space-times near spatial infinity. While Theorem (4.3) shows that the conformal factor $\Omega_{S}$ may be considered as a function of the conformal factor $\Omega_{A}$, the present theorem shows that the metric $h$ must have a very special behaviour at the point $i$. Though these two facts are not needed to demonstrate the existence of an (analytic) conformal extension of the static solution through null infinity, they may be of significance if one seeks a detailed understanding of the point spatial infinity in the 4-dimensional space-time picture.

By Theorem (4.2) the result above implies that the Bach-tensor of the solution $h$ of the conformal static equation (4.4)-(4.9) satisfies the conditions (3.12). This has been noted independently by R. Beig [4].

To prove the theorem, we may use the expression (4.17) for $\Omega_{A}$ and Eqs. (4.4)-(4.9) to check by straightforward calculation that the condition (3.4) is satisfied. For later use it is however more convenient to calculate instead the explicit expression for the tensor $d_{a b}$ derived from $h$ and the conformal factor

$$
\Omega=\omega\left\{\cosh \left(\frac{M_{0}}{2} \sqrt{\omega}\right)+b \sinh \left(\frac{M_{0}}{2} \sqrt{\omega}\right)\right\}^{-2} .
$$

We obtain

$$
d_{a b}=p(\omega) S_{a b}+q(\omega)\left\{D_{a} \omega D_{b} \omega-\frac{1}{3} h_{a b} D^{c} \omega D_{c} \omega\right\}
$$


where, with the notation $z=\left(M_{0} / 2\right) \sqrt{\omega}$, we have

$$
\begin{aligned}
p= & \frac{b M_{0}^{2}}{4 z}\left(\cosh ^{2} z+\sinh ^{2} z\right)+\frac{\left(1+b^{2}\right) M_{0}^{2}}{4} \cosh z \frac{\sinh z}{z}, \\
q= & \frac{b M_{0}^{2}}{8 z^{5}}\left\{4 z \cosh z \sinh z-\left(3+4 z^{2}\right)\left(\cosh ^{2} z+\sinh ^{2} z\right)\right\} \\
& +\frac{\left(1+b^{2}\right) M_{0}^{2}}{8 z^{5}}\left\{3 z\left(\cosh ^{2} z+\sinh ^{2} z\right)-\left(3+4 z^{2}\right) \cosh z \sinh z\right\} .
\end{aligned}
$$

It follows that $d_{a b}=0\left(\omega^{-3 / 2}\right)$ for $\omega \rightarrow 0$ if $b \neq 0$, while the functions $p(\omega), q(\omega)$ are analytic with

$$
\lim _{\omega \rightarrow 0} p(\omega)=\frac{M_{0}^{2}}{4}, \quad \lim _{\omega \rightarrow 0} q(\omega)=-\frac{2 M_{0}^{2}}{15} \text { if } b=0 .
$$

\section{On Radiative Space-Times}

Theorem (4.4) is particularly interesting, because it shows a way to construct radiative initial data sets.

Theorem (5.1). Suppose the metric $h$ and the function $\omega$ are part of an analytic solution of the conformal static field equations (4.4)-(4.9). Then the radiative initial data set derived according to (2.25) from $h$ and the analytic function $\Omega_{A}$ given by (4.17) determines a solution of Einstein's field equation $\operatorname{Ric}[\tilde{g}]=0$ (unique near past time-like infinity) which has a smooth structure at past null infinity and is regular and analytic at past time-like infinity.

The solutions of Einstein's field equations considered in the theorem, which will be referred to as "radiative space-times" in the following, are obtained as follows. The methods and results discussed in [9] imply the existence of a unique solution comprising a metric $g$ and a conformal factor $\Omega$ of the regular conformal field equations on some manifold $M$. The metric $\tilde{g}=\Omega^{-2} g$ on the manifold $I^{+}(i)$ constitutes the radiative space-time. A discussion similar to that given in the case of the hyperboloidal initial value problem [12] shows that past null infinity of the solution $\left(I^{+}(i), \tilde{g}\right)$ is smooth.

There are a few questions which may come up at first sight of Theorem (5.1). We shall discuss some of them now.

One may wonder whether at least on some parts of $M$ where $\Omega \neq 0$ the metric $\tilde{g}=\Omega^{-2} g$ is conformal to a static metric. It is essentially a consequence of Brinkmann's theorem (see [16] and the literature given there) and follows easily from Eqs. (2.2)-(2.6), that this cannot be the case unless $\tilde{g}$ is flat. If we assume that $\hat{g}_{\mu \nu}=\theta^{2} \tilde{g}_{\mu \nu}$ for suitable $\theta>0$ is a static solution of Einstein's vacuum field equations, we may apply the regular conformal field equations to this situation. Since $\hat{R}_{\mu \nu}=0$, Eq. (2.3) implies that $\hat{S}=\frac{1}{4} \hat{\nabla}_{i} \hat{\nabla}^{i} \theta=\hat{s}_{0}=$ const, and therefore by (2.2), (2.4), (2.6),

$$
\begin{aligned}
\hat{\nabla}_{i} \hat{\nabla}_{k} \theta & =\hat{s}_{0} \hat{g}_{i k}, \\
\hat{\nabla}_{i} \theta \hat{\nabla}^{i} \theta & =2 \theta \hat{s}_{0}, \\
\hat{\nabla}_{i} \theta \hat{C}^{i}{ }_{j k l} & =0 .
\end{aligned}
$$


If $\hat{s}_{0} \neq 0$, then $\hat{\nabla}^{i} \theta$ is time- or space-like everywhere by (5.2), and (5.3) implies $C_{j k l}^{i} \equiv 0$ (seen immediately in the spinor formalism). If $\hat{s}_{0}=0$ but $\nabla_{i} \theta \neq 0$ and $C_{j k l}^{i} \neq 0$, then (5.3) would imply that $\hat{g}$ is of Petrov type $N$. However, this cannot be the case if $\hat{g}$ is static. It follows that either $\hat{g}$ is conformally flat or that $\theta=\theta_{0}=$ const, whence $\tilde{g}$ is static. As we shall see below this can only be the case if $\tilde{g}$ is flat.

The question whether there is actually radiation present in the "radiative space-times" discussed here may be settled by looking at the identity (3.8). It shows that unless the metric $h$ is conformally flat the conformal Weyl curvature of the metric $\tilde{g}$ cannot vanish near past time-like infinity. Therefore the radiation field on $\mathscr{I}^{-}$cannot vanish identically near past time-like infinity by the uniqueness result of $[10]$.

As illustrated by the Schwarzschild solution and the discussion of (3.8), this identity does not allow one to conclude that the conformal Weyl curvature of $g$ vanishes on the initial surface if the metric is conformally flat. If, however, $\Omega$ is analytic and the radiativity condition is satisfied at $i$, then the left-hand side of (3.8) vanishes if and only if $d_{a b}$ vanishes near $i$.

It is well known that the assumptions of asymptotic simplicity and of the existence of Killing fields together have very strong implications on the structure of the space-time $[1,2]$. This is likely to be the source of the difficulties in finding exact solutions which represent purely radiative space-times (if these exist). But in the case of Einstein's field equations with positive cosmological constant the implications are equally strong, nevertheless it has been shown that "asymptotic simplicity" describes correctly the asymptotic behaviour in that case $[11,12])$ ). In fact, in the case of the boost-rotation symmetric solutions which are regular at past time-like infinity the generators of $\mathscr{I}^{-}$are not complete [5]. In constructing the radiative solutions as suggested by Theorem (5.1) we loose the static Killing field (see below). However, it may well be that we just trade one symmetry for another, which possibly forces some generators of $\mathscr{I}^{-}$to be incomplete (if the analytic extension of the solution is considered). It is one of the purposes of the following discussion of Killing fields on the radiative space-times to show that this is not necessarily true.

Suppose $\widetilde{K}^{\mu}$ is a Killing vector field for the radiative metric $\tilde{g}_{\mu \nu}$ on $I^{+}(i)$. If we set $K^{\mu}=\widetilde{K}^{\mu}, K_{\mu}=g_{\mu \nu} K^{v}=\Omega^{2} \tilde{g}_{\mu v} \widetilde{K}^{v}$, then with respect to the metric $g$ the field $K$ satisfies the conformal Killing equations

with

$$
\nabla_{i} K_{j}+\nabla_{j} K_{i}=2 \Phi g_{i j}
$$

$$
\Phi \equiv \frac{1}{4} \nabla_{j} K^{j}=\Omega^{-1} K^{j} \nabla_{j} \Omega .
$$

Setting $\Phi_{j}=\nabla_{j} \Phi$, the following conditions are satisfied by the conformal Killing field $K$ (see e.g. [22])

$$
\begin{gathered}
\nabla_{i} \nabla_{j} K_{l}=-K^{p} r_{p i l j}+\Phi_{i} g_{j l}+\Phi_{j} g_{l i}-\Phi_{l} g_{j i} \\
\nabla_{i} \Phi_{j}=-\mathscr{L}_{K}\left(\frac{1}{2}\left\{r_{i j}-\frac{1}{6} r g_{i j}\right\}\right), \\
\mathscr{L}_{K} C^{i}{ }_{j k l}=0
\end{gathered}
$$




$$
\mathscr{L}_{K}\left(\nabla_{[l} r_{i] j}-\frac{1}{6} \nabla_{[l} r g_{i] j}\right)=-\Phi^{k} C_{k j i l},
$$

where $\mathscr{L}_{K}$ denotes the Lie-derivative with respect to $K$.

Equations (5.4), (5.6), (5.7) imply along any given smooth curve an ordinary linear homogeneous differential equation for the "conformal Killing data" $K_{j}, \Phi$, $\Phi_{i j}=\nabla_{[i} K_{j]}, \Phi_{j}$. This implies that the field $K$ is analytic near past time-like infinity and may in fact be extended to an analytic conformal Killing field on a neighbourhood of the point $i$. We will again denote this extension by $K$.

Since $K$ is a Killing vector field for the physical metric on $I^{+}(i)$, its flow maps the past endpoints of the physical null geodesics, represented by the points of $\mathrm{N}_{i}^{+}$ again into such endpoints. Therefore $K$ is tangent to $N_{i}$. In particular we have

$$
K=0, \quad \Phi=0 \text { at } i .
$$

This may also be deduced formally by evaluating (5.5) on $\{\Omega=0\}$ and the first and second covariant derivative of (5.5) at $i$. By (5.10) the freedom to give conformal Killing data is reduced to the dimension of the Poincare group.

As mentioned before, we have

Theorem (5.2). If a radiative space-time admits a time-like Killing vector field, it is flat.

If $\gamma$ is a null geodesic for $g$, then the scalar product $g\left(\gamma^{\prime}, K\right)$ is constant along $\gamma$. Since $K$ is time-like on $I^{+}(i)$, it follows that $K \neq 0$ at all points of $M$ which can be reached from $I^{+}(i)$ by a null geodesic. In particular $K \neq 0$ on $N_{i}{ }^{+} \backslash$ i. Choosing normal coordinates $x^{j}=\delta^{j}{ }_{\mu} x^{\mu}$ and a normal frame $e^{\mu}{ }_{k}\left(\partial / \partial x^{\mu}\right)$ (with $e^{\mu}{ }_{k}=\delta^{\mu}{ }_{k}$ at $\left.i\right)$ centered at $i$, we may expand $K^{j}$ in the form considered in (4.15). In view of (5.10), (5.6) this gives

$$
K^{j}\left(x^{p}\right)=x^{l} \nabla_{l} K^{j}(i)+\left(x^{l} \Phi_{l}(i) x^{j}-\frac{1}{2} x^{l} x_{l} \Phi^{j}(i)\right)+O\left(|x|^{3}\right) .
$$

Since $K^{j}$ is time-like on $I^{+}(i)$ and tangent to $N_{i}{ }^{+}$, it must be null on $N_{i}{ }^{+}$. If $l^{j} \neq 0$ is a future directed null vector at $i$ it follows that

$$
K^{j}\left(\lambda l^{p}\right)=f(\lambda) l^{j} \text { for } \lambda \geqq 0
$$

with some analytic function $f$, which may depend on $l$, such that $f(\lambda)>0$ for $\lambda>0, f(0)=0$ (assuming $K$ as future directed on $I^{+}(i)$ ). Expanding $f(\lambda)=$ $f_{1} \lambda+f_{2} \lambda^{2}+O\left(\lambda^{3}\right)$ we find from (5.11), (5.12),

$$
\begin{aligned}
& f_{1} l^{j}=l^{k} \nabla_{k} K^{j}(i), \\
& f_{2} l^{j}=l^{k} \Phi_{k}(i) l^{j} .
\end{aligned}
$$

By (5.4), (5.10) $\nabla^{l} K_{j}(i)$ is a generator of the Lorentz group, which by (5.13) has any null vector as an eigenvector, whence

$$
\nabla_{l} K_{j}=0 \text { at } i, \quad f_{1}=0 .
$$

Since $\Phi_{j}(i)$ is the remaining conformal Killing datum of $K$ at $i$ and $K_{j} K^{j}<0$ on $I^{+}(i)$, we conclude in view of (5.11)

$$
\Phi_{j}(i) \neq 0, \quad \Phi_{j} \Phi^{j} \leqq 0 \text { at } i, \quad f_{2}=l^{k} \Phi_{k}(i) .
$$


We shall now show that Eq. (5.8), which can be rewritten

$$
\mathscr{L}_{K}\left(d_{i j k l}\right)=\Phi d_{i j k l},
$$

implies that the null datum for $d_{i j k l}$ on $N_{i}$ vanishes. Choose $l^{k}$ as before with $l^{k} \Phi_{k}(i) \neq 0$. At a point $\gamma\left(\lambda_{0}\right), \lambda_{0}>0$ of the generator $\gamma^{k}(\lambda)=\lambda l^{k}$ of $N_{i}{ }^{+}$choose vectors $V^{l}, W^{l}$ tangent to $N_{i}^{+}$such that

$$
g(V, V)=g(W, W) ; g(V, W)=0 ; V, W, K \text { are linear independent, }
$$

and extend the fields $V, W$ along $\gamma$ by requiring $\mathscr{L}_{K} V=0, \mathscr{L}_{K} W=0$. The relations (5.18) will then be preserved along $\gamma$ since $K$ is a conformal Killing vector field. Set $M^{j}=V^{j}+\sqrt{-1} W^{j}$. Up to a non-vanishing factor the null datum of the field $d_{i l j k}$ is given by $d=d_{i l j k} K^{i} M^{l} K^{j} M^{k}$ at $\gamma(\lambda), \lambda>0$. Equations (5.17), (5.12) imply along $\gamma(\lambda), \lambda>0$

$$
f(\lambda) \frac{d}{d \lambda} d=\Phi d
$$

which may be rewritten, using (5.10), (5.15), (5.16) in the form

$$
\frac{d}{d \lambda}\left(\lambda^{-1} d\right)=h(\lambda)\left(\lambda^{-1} d\right)
$$

with some function $h(\lambda)$ which is continuous for $\lambda \geqq 0$. Since $M^{j}=O(\lambda), K^{j}=O(\lambda)$ for $\lambda \rightarrow 0$ the function $\lambda^{-1} d$ extends to a $C^{1}$ function of $\lambda$ for $\lambda \geqq 0$, which vanishes at $\lambda=0$ and thus by the last equation everywhere on the generator $\gamma$. Since the null datum of $d_{i j k l}$ vanishes on $N_{i}^{+}$, the metric $\tilde{g}$ on $I^{+}(i)$ is flat by the uniqueness result of $[10]$.

The question whether the radiative solutions admit necessarily a Killing vector field finds an answer by

Theorem (5.3). Let $S_{a b}$ be the traceless part of the Ricci tensor of the metric $h$ considered in Theorem (5.1). If the corresponding space spinor field is not of the algebraically special form.

$$
S_{A B C D}=\alpha_{(A} \alpha_{B} \beta_{C} \beta_{D)} \text { at } i
$$

with some spinors $\alpha_{C}, \beta_{C}$, and satisfies

$$
D_{(A B} S_{C D E F)}=0 \text { at } i,
$$

the radiative space-time determined from $h$ admits no Killing field.

Remark. The conditions on $S_{a b}$ stated here are conditions on the first free data in the characterization discussed in Theorem (4.2) and leave open the choice of an infinite number of free data. Since we can have real space spinors of the form $S_{A B C D}=\alpha_{(A} \beta_{B} \gamma_{C} \delta_{D)}$ with pairwise linear independent spinors $\alpha_{A}, \beta_{B}, \gamma_{C}, \delta_{D}$, this suggests that the class of radiative space-times without any symmetry is indeed quite large.

To prove our assertion let $\varphi_{A B C D}$ be the rescaled Weyl spinor which is the (space-time) spinor representation of the tensor $d_{i j k l}$. Since $d_{0 a b c} \equiv 0$ on $S$ and 
$d_{0 a 0 b}=M_{0}^{2} / 4 S_{a b}$ at $i$ by $(4.19,4.20)$, we have

$$
\varphi_{A B C D}=\frac{M_{0}^{2}}{4} S_{A B C D} \text { at } i \text {. }
$$

Because of (5.10), Eq. (5.17) reduces at the point $i$ to an algebraic relation for the tensors $d_{i j k l}$ and $\nabla_{i} K_{j}$. Expressed in terms of space-time spinors it reads in view of (5.21),

$$
\varphi^{F}{ }_{(A} S_{B C D) F}=0 \text { at } i,
$$

where $\varphi_{A B} \equiv \frac{1}{2} \nabla_{A F^{\prime}} K_{B}{ }^{F^{\prime}}$ is symmetric because of (5.10). Writing $\varphi_{A B}=\psi_{A B}+i \rho_{A B}$ with (uniquely determined) real space spinors $\psi_{A B}, \rho_{A B}$ and observing that $S_{A B C D}$ is a real space spinor, Eq. (5.22) splits into

$$
\psi^{F}{ }_{(A} S_{B C D) F}=0, \quad \rho^{F}{ }_{(A} S_{B C D) F}=0 \text { at } i .
$$

If $\psi_{A B}$ has components $\psi_{00}=0, \psi_{11}=0, \psi_{01}=\psi_{10}=1$ one finds by direct calculation that the first of the relations (5.23) can only be satisfied if $S_{A B C D}=$ $c \alpha_{(A} \alpha_{B} \beta_{C} \beta_{D)}$ with some $c \in \mathbb{C}$ and spinors $\alpha_{A}, \beta_{B}$ with components $\alpha_{0}=\beta_{1}=0$, $\alpha_{1}=\beta_{0}=1$. Since the group $S U(2)$ acts transitively on the directions at $i$, the relations (5.23) can always be reduced to this case unless $\psi_{A B}=0, \rho_{A B}=0$. By the assumptions of the theorem it follows that necessarily $\nabla_{j} K_{l}=0$ at $i$ and the remaining conformal Killing datum at $i$ is given by $\Phi_{j}$. By (5.6) the second covariant derivative is determined by $\Phi_{j}$ at $i$. From (5.20), (4.8), (4.9) we get $D_{c} S_{a b}=0$ at $i$, which allows one with (4.19), (4.20) to conclude that $D_{c} d_{a b}=0$ at $i$. Therefore the evaluation of the conformal vacuum Bianchi identity (2.5) at $i$ yields in view of (2.23), (2.24) that $\nabla_{i} d_{j k l m}=0$ at $i$. Consequently one obtains an algebraic relation for $\Phi_{k}$ and $d_{i j k l}$ if one takes a covariant derivative of (5.17) and evaluates at $i$. From this we get the spinor relation

$$
3 \varphi_{A B C D} \Phi_{E E^{\prime}}+2 \varepsilon_{E(A} \varphi_{B C D) F} \Phi_{E^{\prime}}^{F}+2 \varphi_{E(A B C} \Phi_{D) E^{\prime}}=0 \text { at } i
$$

which upon contraction with $\varepsilon^{D E}$ yields

$$
S_{A B C D} \Phi_{E^{\prime}}^{D}=0 \text { at } i \text {. }
$$

Since we have forbidden the form (5.19) this cannot be true if $\Phi_{A A^{\prime}}=\gamma_{A} \bar{\gamma}_{A^{\prime}} \neq 0$. However $\Phi^{A A^{\prime}} \Phi_{A A^{\prime}} \neq 0$ would imply $S_{A B C D}=0$ at $i$ which contradicts our assumptions.

Acknowledgements. I should like to thank the members of the relativity group in Pittsburgh for hospitality and discussions at an early stage of this work. This work has been supported by a Heisenberg-fellowship of the Deutsche Forschungsgemeinschaft.

\section{References}

1. Ashtekar, A., Xanthopoulos, B.C.: Isometries compatible with asymptotic flatness at null infinity: A complete description. J. Math. Phys. 19, 2216-2222 (1978)

2. Ashtekar, A., Schmidt, B. G.: Null inifinity and Killing fields. J. Math. Phys. 21, 862-867 (1980)

3. Beig, R., Simon, W.: Proof of a multipole conjecture due to Geroch. Commun. Math. Phys. 79, $581-589(1981)$ 
4. Beig, R.: private communication

5. Bicak, J., Schmidt, B. G.: Isometries compatible with gravitational radiation. J. Math. Phys. 25, 600 (1984)

6. Choquet-Bruhat, Y., York, J. W.: The Cauchy problem. In: General relativity and gravitation. Held, A. (ed.). New York: Plenum Press 1980

7. Einstein, A.: Demonstration of the non-existence of gravitational fields with a non-vanishing total mass free of singularities. Univ. Nac. Tucuman, Revista A 2, 5-15 (1941)

8. Friedrich, H.: The asymptotic characteristic initial value problem for Einstein's vacuum field equations as an initial value problem for a first-order quasilinear symmetric hyperbolic system. Proc. R. Soc. Lond. A 378, 401-421 (1981)

9. Friedrich, H.: Cauchy problems for the conformal vacuum field equations in General Relativity. Commun. Math. Phys. 91, 445-472 (1983)

10. Friedrich, H.: On purely radiative space-times. Commun. Math. Phys. 103, 35-65 (1986)

11. Friedrich, H.: Existence and structure of past asymptotically simple solutions of Einstein's field equations with positive cosmological constant. J. Geom. Phys. 3, 101-117 (1986)

12. Friedrich, H.: On the existence of $n$-geodesically complete or future complete solutions of Einstein's field equations with smooth asymptotic structure. Commun. Math. Phys. 107, 587-609 (1986)

13. Friedrich, H., Schmidt, B. G.: Conformal geodesics in general relativity. Proc. R. Soc. A 414, 171-195 (1987)

14. Garabedian, P.: Partial differential equations. New York: J. Wiley 1964

15. Geroch, R.: Multipole moments. II. Curved Space. J. Math. Phys. 11, 2580-2588 (1970)

16. Halford, W. D.: Brinkmann's theorem in general relativity. GRG 14, 1193-1195 (1982)

17. Kodaira, K.: Complex manifolds and deformation of complex structure. Berlin, Heidelberg, New York: Springer 1986

18. Penrose, R., Rindler, W.: Spinors and space-time, Vol. 1. Cambridge: University Press 1984

19. Reula, O.: On existence and behaviour of asymptotically flat solutions to the stationary Einstein equations. Submitted to Commun. Math. Phys

20. Schoen, R., Yau, S. T.: On the proof of the positive mass conjecture in General Relativity. Commun. Math. Phys. 65, 45-76 (1976)

21. Sommers, P.: Space spinors. J. Math. Phys. 21, 2567-2571 (1980)

22. Yano, K.: The theory of Lie derivatives and its applications. Amsterdam: North Holland 1957

23. Zipoy, D. M.: Topology of some spheroidal metrics. J. Math. Phys. 7, 1137-1143 (1966)

Communicated by L. Alvarez-Gaumé

Received February 10, 1988; in revised form April 20, 1988 
